\title{
Updating toward the signal
}

\author{
Christopher P. Chambers • Paul J. Healy
}

Received: 5 February 2010 / Accepted: 19 November 2010 / Published online: 4 December 2010 (C) The Author(s) 2010. This article is published with open access at Springerlink.com

\begin{abstract}
Modelers frequently assume (either implicitly or explicitly) that an agent's posterior expectation of some variable lies between their prior mean and the realization of an unbiased signal of that variable. We call this property updating toward the signal (UTS). We show that if the prior and signal error densities are both symmetric and quasiconcave then UTS will occur. If, for a given prior, UTS occurs for all symmetric and quasiconcave error densities, then in fact the prior must be symmetric and quasiconcave. Similar characterizations are derived for two additional updating requirements that are strictly weaker than UTS.
\end{abstract}

Keywords Signal extraction - Bayes's rule - Reversion to the mean . Posterior beliefs · Bayesian robustness

JEL Classification $\quad$ C11 D D 1 D D 1 D D3 - D84

\section{Introduction}

It is common in economic theory to assume that agents infer the value of some meaningful random variable based on imperfect observation. For example, bidders

The authors thank Federico Echenique, Laurent Mathevet, Jim Peck, Larry Samuelson, and Leonard Stefanski for their valuable comments, and The Little River Band for their inspiration.

C. P. Chambers $(\bowtie)$

Department of Economics, University of California, San Diego, 500 Gilman Drive 0508, La Jolla, San Diego, CA 92093-0508, USA

e-mail: cpchambers@dssmail.ucsd.edu

\section{P. J. Healy}

Department of Economics, The Ohio State University, 1945 North High Street, Columbus, $\mathrm{OH} 43210$, USA 
in common-value auctions infer the unknown value of an object from private signals (Wilson 1977; Milgrom and Weber 1982), oligopolists choose production levels after observing noisy signals of the demand curve or of their marginal costs (Novshek and Sonnenschein 1982; Shapiro 1986), producers in the Lucas "island" economy react to prices that serve as noisy signals of aggregate demand (Lucas 1972), and agents search for the best price or wage when the underlying distribution is unknown (DeGroot 1968; Rothschild 1974).

A standard intuition in such settings is that, by Bayes's rule, posterior beliefs should represent a weighted average of the prior beliefs and the observed signal. Thus, it is often assumed that the posterior expectation lies between the prior expectation and the observed signal for every possible signal value. We refer to this property as updating toward the signal (UTS). UTS holds for standard examples such as a normal prior with a normally distributed and independent signal error. In general, however, UTS does not hold; Observation 1 below shows how any arbitrary combination of a prior mean, signal realization, and posterior mean can occur when no restrictions are placed on the distributions.

Although many authors implicitly assume UTS by analyzing only normally distributed random variables (Grossman and Stiglitz 1976, 1980; Morris and Shin 2002, 2006), some make this assumption explicit (Shapiro 1986; Moore and Healy 2008) as it is necessary for the results they describe. UTS clearly imposes restrictions on the possible distributions of the variable and the signal error. To the best of our knowledge, however, the nature of these restrictions are not understood.

In addition to UTS, we also study the weaker updating requirements of updating in the direction of the signal (UDS), where the posterior mean must lie in the same direction as the signal (relative to the prior mean), and mean reinforcement (MR), which requires that the posterior mean equal the prior mean when the signal equals the prior mean. We find that the necessary and sufficient conditions for UTS can be weakened in intuitive ways to provide necessary and sufficient conditions for UDS and MR as well.

Throughout this paper, we allow the error to depend on the realizations of the underlying variable; however, we require that this dependence be symmetric in the underlying variable. In other words, we require that the signal error distributions be identical for any two realizations of the underlying variable that are equidistant from the prior mean. We refer to this condition as symmetric dependence. Under symmetric dependence, we find that MR obtains when the prior distribution and the (mean-zero) error distribution are both symmetric. If the error term is also quasiconcave, then UDS is guaranteed. If, in addition, the errors are independent and the prior is quasiconcave, then UTS is satisfied. We argue that these results are tight by showing examples where the conditions for one updating requirement (e.g., UDS) are satisfied, but the stronger updating requirement (e.g., UTS) fails.

In the other direction, one would like to know the content of explicitly assuming UTS (or UDS or MR) in a theoretical model. To examine this, we take the approach of a modeler who is free to choose a prior distribution but cannot commit to strong assumptions about the error distribution. This would be appropriate, for example, in a model of asset returns with market microstructure noise because the exact cause 


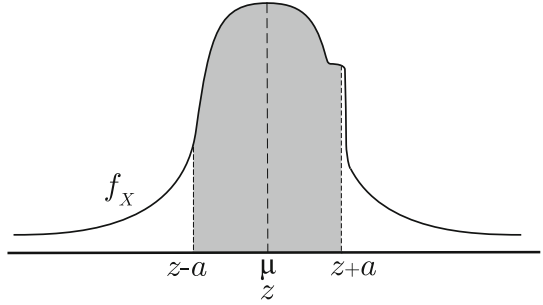

(A)

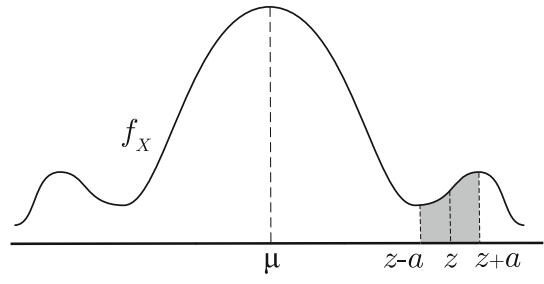

(B)

Fig. 1 Demonstrating the necessary conditions for a MR and UDS, and b UTS

and structure of the noise in high-frequency asset return data is not well understood. $^{1}$

Assuming that the family of admissible error distributions is a broad enough class of symmetric distributions (specifically, assuming the family contains all symmetric distributions that put a mass of one half on two different mass points), we show that if MR holds for all errors in this family, then the prior must be symmetric. If the stronger condition of UDS is assumed, then an impossibility result obtains: there is no prior distribution for which UDS can be guaranteed for all errors in the family. If the family of errors is a broad enough class of symmetric and quasiconcave distributions (specifically, assuming the family contains all symmetric uniform distributions), we show that MR again implies a symmetric prior, but now UDS places no additional restrictions; the two are equivalent assumptions. Finally, assuming UTS in this case implies that the prior must be both symmetric and quasiconcave.

Figure 1 provides a rough intuition for why symmetry is necessary for MR and UDS, and why quasiconcavity is also necessary for UTS. In Fig. 1a, we show an asymmetric prior density $f_{X}$ with mean $\mu$. If the error term has a uniform distribution with radius $a>0$, then the posterior distribution after observing a signal $z=\mu$ is given by the shaded area under the prior density function. ${ }^{2}$ The asymmetry in the prior leads to an asymmetry in the posterior. In the figure, this causes the posterior mean to be slightly greater than the prior mean. MR is then violated because a signal equal to the prior mean leads to a posterior mean that is different from the prior mean. If $z$ were slightly less than $\mu$, then the posterior mean would still be greater than $\mu$, and now UDS would be violated since the signal is less than the prior mean while the posterior mean is greater. Following this argument for every $a>0$ leads to the conclusion that MR and UDS require a symmetric prior density function when all uniformly distributed errors are admissible.

\footnotetext{
1 Zhou (1996), Andersen et al. (2000), and others show that, in practice, this microstructure noise creates a substantial bias when estimating the volatility of an asset using the realized variance of returns sampled at a high frequency. In response, Zhang et al. (2005), Hansen and Lunde (2006), and Aït-Sahalia et al. (2005) explicitly model the microstructure noise as a random error term added to the underlying asset price - acknowledging that the structure of this noise is not well understood-and then derive the optimal estimation strategy under these conditions.

2 Technically, the posterior distribution would be normalized to have a unit integral.
} 
In Fig. 1b, we show a prior distribution that is symmetric, but not quasiconcave. Again, suppose the error distribution is uniform with radius $a>0$ so that the posterior distribution after observing the signal $z$ is given by the shaded area under the curve. Since the posterior distribution in this example is strictly increasing over its domain, its mean must be greater than $z$. This violates UTS because the posterior mean is farther from the prior mean than the signal. In general, if the signal realization $z$ occurs in a region where quasiconcavity of the prior fails and if the radius of the uniform error distribution is sufficiently small, then UTS will be violated. Thus, quasiconcavity is necessary for UTS when all uniformly distributed errors are admissible.

These results lead to three characterizations. First, if the family of error terms is a sufficiently broad class of symmetric errors, then symmetry of the prior is equivalent to requiring MR for the entire family of errors. Second, if the family of error terms is a sufficiently broad class of symmetric and quasiconcave error terms, then symmetry of the prior is equivalent to MR and MR is equivalent to UDS. Finally, for the same family of symmetric and quasiconcave error terms, symmetry and quasiconcavity of the prior is equivalent to UTS.

In econometrics, since the posterior mean can also be thought of as the Bayes estimator of the underlying variable using a quadratic loss function, the sufficient conditions for UTS can be also used to guarantee reasonably well-behaved parameter estimates without relying on the standard normality assumptions. For example, the literature on robust Bayesian analysis (see Berger 1994 for an overview) argues that the family of symmetric and quasiconcave prior densities is particularly robust when performing Bayesian hypothesis tests; since we show that symmetric and quasiconcave priors imply and are implied by UTS (when the error distributions are also symmetric and quasiconcave), we provide another sense in which this family of priors is robust. ${ }^{3}$

Perhaps the closest paper to ours is the work of Andrews et al. (1972). Using a normally distributed error distribution and a symmetric prior, they show that mean reinforcement (MR) is satisfied and that if the prior is also quasiconcave then UTS is satisfied. ${ }^{4}$ Since normal densities are symmetric and quasiconcave, these two results are corollaries of our Propositions 1 and 3, respectively.

In the economics literature, this paper is similar in spirit to Milgrom (1981). Milgrom requires that higher signal realizations lead to higher posterior beliefs in the sense of first-order stochastic dominance. He uses a similar robustness approachrequiring monotonicity for all non-degenerate priors-and shows that a signal distribution has the strict monotone likelihood ratio property (MLRP) if and only if monotonicity holds for all priors. Our paper differs from Milgrom's in that we seek to

\footnotetext{
3 There has also been some interest by statisticians in credibility theory - the use of posterior means that are (typically) assumed to be linear in the observed data to adjust parameter estimates in actuarial models-and its appropriateness (see Kahn 1975 for an introduction). The capstone of this literature is Diaconis and Ylvisaker (1979), in which the authors characterize a conjugate family of distributions by requiring linearity of the posterior expectation. Our results are independent of this literature, however, since linearity of the posterior expectation is neither implied by nor implies UTS. Furthermore, the statistics literature has focused on implied restrictions on the parameters of certain families of distributions rather than restrictions on the density function directly, as provided by our results.

4 This work is followed by Polson (1991), who provides an exact representation of the posterior mean for the case of normally distributed errors and arbitrary priors.
} 
order the posterior mean relative to the prior mean while Milgrom's goal is to order various posterior distributions according to their signal realizations; in fact, the UTS concept is neither implied by nor implies any sort of monotonicity of the posterior mean. Additionally, Milgrom's approach fixes an error distribution and considers the family of all non-degenerate priors, while we fix a prior and consider various families of error distributions. A systematic study of stochastic dominance in the latter case has not been undertaken; however, we show in a related paper (Chambers and Healy 2009) that for any fixed prior with bounded support one can find a symmetric, quasiconcave error distribution and a pair of signals such that the lower signal generates a posterior that strictly stochastically dominates the posterior formed from the higher signal. Thus, one can always reverse (and not just negate) Milgrom's monotonicity result by finding an error term that does not satisfy MLRP but is well behaved in many other respects.

Finally, work which is related in a broader sense includes Feldman (1991), Jackson and Peck (1999), Ghirardato (2002), Tadelis (2003), Sandroni (2005), Azrieli and Lehrer (2007), Menon and Subramanian (2008), and Quint (2010). ${ }^{5}$

We introduce the environment and notation in Sect. 2. Results appear in Sect. 3, which is broken into three subsections. The first covers the sufficiency results, the second lists the necessity results, and the third combines these into three characterization theorems. In Sect. 4, we show how the characterization theorems can be applied in the context of a portfolio choice problem.

\section{Environment and notation}

We study situations in which a decision maker is interested in the realization of some underlying real-valued random variable $X$ but instead observes a realization of a real-valued random variable $Z$ that serves as a signal of $X$. Formally, consider two probability spaces $(\Omega, \mathcal{B}, \mu)$ and $\left(\Omega^{\prime}, \mathcal{B}^{\prime}, \mu^{\prime}\right)$. Let $X: \Omega \rightarrow \mathbb{R}$ be the underlying random variable of interest, and, for every $x \in \mathbb{R}$, let $\tilde{\varepsilon} \mid x: \Omega^{\prime} \rightarrow \mathbb{R}$ be another random variable. Together, these random variables jointly generate a random variable $Z: \Omega \times \Omega^{\prime} \rightarrow \mathbb{R}$ given by

$$
Z\left(\omega, \omega^{\prime}\right)=X(\omega)+[\tilde{\varepsilon} \mid X(\omega)]\left(\omega^{\prime}\right) .
$$

We assume below that each $\tilde{\varepsilon} \mid x$ has a mean of zero $(E[\tilde{\varepsilon} \mid x]=0)$, so that $Z$ represents an unbiased signal of the realization $X(\varepsilon)$. In this case, each variable $\tilde{\varepsilon} \mid x$ is referred to as an error term. Let $\{\tilde{\epsilon} \mid x\}_{x}$ denotes a collection of error terms, one for each $x \in \mathbb{R} .^{6}$

\footnotetext{
5 These references were added at the suggestion of an anonymous referee.

6 Alternatively, one could start with $X$ and $Z$ as primitives and then derive the conditional distributions $X \mid Z$. This approach faces the problem that conditional distributions and conditional expectations are not unique as they may differ on probability-zero events. The family of conditional distributions we use here as primitive could be thought of as one particular family of possible conditional distributions derived from some underlying variables $X$ and $Z$.
} 
We can now define the 'unconditional' error term $\tilde{\varepsilon}\left(\omega, \omega^{\prime}\right)=(\tilde{\varepsilon} \mid x(\omega))\left(\omega^{\prime}\right)$, so that $Z=X+\tilde{\varepsilon}$, where we also understand $X$ to be a random variable defined on $\Omega \times \Omega^{\prime}$ but constant on $\Omega^{\prime}$. Thus, properties of $\tilde{\varepsilon}$ can equivalently be thought of as properties of the family $\{\tilde{\varepsilon} \mid x\}_{x}$, and vice-versa. We emphasize that writing the signal in the additive form $(Z=X+\tilde{\varepsilon})$ does not assume independence between $\tilde{\varepsilon}$ and $X$; for example, a multiplicative error structure of the form $Z=X \tilde{\theta}$, where $\tilde{\theta}$ is independent of $X$ (as in Lucas 1972), can be rewritten as an additive structure by defining $\tilde{\varepsilon}=X \tilde{\theta}-X$ (or $\tilde{\varepsilon} \mid x=x \tilde{\theta}-x$ for each $x$ ), which clearly depends on $X$.

Realizations of $X, Z$, and $\tilde{\varepsilon}$ are represented by $x, z$, and $\varepsilon$, respectively. For each possible realization $z$ of the signal $Z$, the decision maker forms a posterior belief about $X$ via Bayes's rule, represented by the random variable $X \mid z$. The goal of our study was to identify conditions on the distributions of $X$ and $\tilde{\varepsilon}$ that guarantee updating toward the signal (UTS), which we formally define below. Conversely, we explore the content of assuming that UTS holds for a given family of models. We also perform these inquiries for updating in the direction of the signal (UDS) and mean reinforcement (MR), also defined below.

If each $\tilde{\varepsilon} \mid x$ has a continuous density, then we define the conditional density by

$$
f_{X \mid z}(x \mid z)=\frac{f_{\tilde{\varepsilon}}(z-x \mid x) f_{X}(x)}{\int_{t} f_{\tilde{\varepsilon}}(z-t \mid t) f_{X}(t) \mathrm{d} t}
$$

whenever this ratio exists, and $E[X \mid z]=\int_{x} x f_{X \mid z}(x \mid z) \mathrm{d} x$ is the conditional expectation of $X$ given $Z=z$. In general, if $\tilde{\varepsilon}$ does not have a density, then we define conditional expectations in the usual way. In this case, both $f_{X \mid z}$ and $E[X \mid z]$ need not be unique; different versions of these objects are equivalent if they vary only on sets of realizations of $Z$ that occur with zero probability. Thus, all of our results on conditional expectations and distributions are true 'almost surely' in $Z$.

Throughout this paper, we apply the following assumptions unless otherwise specified:

A1: All random variables have finite means.

A2: The random variable $X$ has a well-defined density function continuous on $\mathbb{R}$.

A3: $E[\tilde{\varepsilon} \mid x]=0$ for each $x$.

The first assumption does rule out some interesting distributions; however, the questions we ask are meaningless if means do not exist, so this assumption is necessary for our inquiry.

The requirement that $X$ has a continuous density function is not substantive but simplifies the presentation of proofs. In several specific examples, we use discontinuous distributions for $X$; in all such cases, the distribution could be replaced by a continuous approximation with no change to the results of the examples.

The third assumption restricts attention to the case of conditionally unbiased signalling structures. Since a Bayesian decision maker must 'de-bias' any observations of biased signals by shifting their posterior beliefs appropriately, properties such as UTS will obviously be violated when this de-biasing generates a large enough shift in posterior beliefs; hence, we only consider the case of conditionally unbiased signals. 
Our sufficiency results are proved assuming each $\tilde{\varepsilon} \mid x$ also has a well-defined density and the collection $\{\tilde{\varepsilon} \mid x\}_{x}$ satisfies a 'symmetric dependence' condition, defined below. Our necessity results make use of error terms with discontinuous densities that are independent of $X$. When we combine these results to generate characterizations, we simply apply continuity arguments to 'close the gap' between these differing assumptions.

The formal definitions of UTS, UDS, and MR are as follows:

Definition 1 Given a random variable $X$ and an error term $\tilde{\varepsilon}$, when $E[X]$ is in the support of $Z$, the pair $(X, \tilde{\varepsilon})$ satisfies mean reinforcement $(M R)$ if

$$
E[X \mid z=E[X]]=E[X]
$$

For continuous distributions, the probability of $E[X]$ occurring is zero, and so $E[X \mid z=E[X]]$ is not uniquely defined. In this case, the definition of mean reinforcement makes sense when we use the specific conditional expectation defined by Eq. 1 .

Definition 2 Given a random variable $X$ and an error term $\tilde{\varepsilon}$, the pair $(X, \tilde{\varepsilon})$ updates in the direction of the signal (or, satisfies $U D S$ ) if for almost every $z$ in $\mathbb{R}$ there exists some $\alpha \geq 0$ such that

$$
E[X \mid z]=\alpha z+(1-\alpha) E[X]
$$

The order of quantification is important in the definition of UDS; because $\alpha$ can vary with $z$, UDS does not require that the posterior mean be linear in the signal realization. See Diaconis and Ylvisaker (1979) for a discussion of the more-restrictive linear case.

Definition 3 Given a random variable $X$ and an error term $\tilde{\varepsilon}$, the pair $(X, \tilde{\varepsilon})$ updates toward the signal (or, satisfies UTS) if for almost every $z$ in $\mathbb{R}$ there exists some $\alpha \in[0,1]$ such that (2) holds.

The two properties of density functions that are central to our results are symmetry and quasiconcavity: ${ }^{7}$

Definition 4 A random variable with cdf $F$ and mean $\mu$ is symmetric if, for every $a \geq 0, F(\mu+a)=1-\lim _{x \rightarrow a^{-}} F(\mu-x)$.

Note that if a symmetric random variable has a continuous density function with finite mean $\mu$, then the density function is symmetric about the mean $(f(\mu+a)=$ $f(\mu-a)$ for all $a)$. Symmetry of $\tilde{\varepsilon}$ is taken to mean symmetry of each $\tilde{\varepsilon} \mid x$.

Definition 5 A random variable is quasiconcave if it has a density function $f$ such that for all $x, x^{\prime} \in \mathbb{R}$ and $\lambda \in(0,1), f\left(\lambda x+(1-\lambda) x^{\prime}\right) \geq \min \left\{f(x), f\left(x^{\prime}\right)\right\}$.

\footnotetext{
${ }^{7}$ Quasiconcave density functions are often called "unimodal" in the statistics literature.
} 
Our results indicate links between quasiconcave and symmetric random variables and UTS, UDS, and MR. Although quasiconcavity and symmetry are strong requirements, they are properties of naturally occurring processes where small deviations from the mean are more frequent than large deviations.

In the following section, we study sufficient conditions on the distributions of $X$ and $\tilde{\varepsilon}$ to guarantee that either UTS, UDS, or MR hold for the posterior distribution, and conversely, what properties of $X$ and $\tilde{\varepsilon}$ are implied by assuming that UTS, UDS, or MR hold for the posterior when a large family of error terms is considered admissible.

\section{Results}

We first verify that Bayes's rule places no restrictions on the location of the posterior mean when no restrictions are placed on the underlying distributions. Thus, restrictions on the pair $(X, \tilde{\varepsilon})$ are necessary to generate restrictions on the relative locations of the signal realization and the prior and posterior means.

Observation 1 For any three real numbers $\mu$, $z$, and $a$, there exist distributions for $X$ and $\tilde{\varepsilon}$ such that $\tilde{\varepsilon}$ is symmetric and independent of $X, E[X]=\mu$ and $E[X \mid z]=a$.

Proof Consider the case of $z \neq a .{ }^{8}$ Pick any continuous $f_{X}$ with $f_{X}(a)>0, f_{X}(2 z-$ $a)=0$, and $\int x f_{X}(x) \mathrm{d} x=\mu$. Let $\tilde{\varepsilon}$ have the two-point distribution $(z-a, 1 / 2 ; a-$ $z, 1 / 2$ ) (meaning $\varepsilon=z-a$ and $\varepsilon=a-z$ each occur with probability one-half), which is independent of $X$. In this case,

$$
E[X \mid z]=a \frac{f_{X}(a)}{f_{X}(a)+f_{X}(2 z-a)}+(2 z-a) \frac{f_{X}(2 z-a)}{f_{X}(a)+f_{X}(2 z-a)}
$$

which then reduces to $E[X \mid z]=a$.

\subsection{Sufficient conditions}

All of the sufficiency results in this section depend on symmetry of the joint distribution $f_{X, \tilde{\varepsilon}}(x, \varepsilon)$ along the $x$-axis in $(x, \varepsilon)$-space, so that, if $X$ is mean-zero, then $f_{X, \tilde{\varepsilon}}(x, \varepsilon)=$ $f_{X, \tilde{\varepsilon}}(-x, \varepsilon)$ for almost all $(x, \varepsilon)$ pairs. Since $f_{X, \tilde{\varepsilon}}(x, \varepsilon)=f_{X}(x) f_{\tilde{\varepsilon} \mid x}(\varepsilon \mid x)$, symmetry is achieved when $f_{X}$ is symmetric and when $f_{\tilde{\varepsilon} \mid x}(\varepsilon \mid x)=f_{\tilde{\varepsilon} \mid x}(\varepsilon \mid-x)$ for every realization $\varepsilon$. This second condition places the reasonably mild restriction on the structure of the dependence of the error term that the error distribution is identical for two realizations of $X$ that are equidistant from its mean. Clearly, error terms that are independent of $X$ satisfy this restriction.

\footnotetext{
${ }^{8}$ If $z=a$, simply let $\tilde{\varepsilon}$ be uniformly distributed with support $[-1,1]$ (independent of $X$ ), and let $X$ have a uniform distribution whose support includes $[a-1, a+1]$ so that $E[X \mid z=a]=\int_{a-1}^{a+1} x(1 / 2) \mathrm{d} x$, which equals $a$.
} 
Definition 6 An error term $\tilde{\varepsilon}$ satisfies symmetric dependence with respect to $X$ if, for each $x, \tilde{\varepsilon} \mid x$ has a continuous density function $f_{\tilde{\varepsilon} \mid x}$ satisfying

$$
f_{\tilde{\varepsilon} \mid x}(\varepsilon \mid E[X]+a)=f_{\tilde{\varepsilon} \mid x}(\varepsilon \mid E[X]-a)
$$

for almost every $\varepsilon$ and $a$ in $\mathbb{R}$.

For notational simplicity, we denote $f_{\tilde{\varepsilon} \mid x}(\varepsilon \mid x)$ by $f_{\tilde{\varepsilon}}(\varepsilon \mid x)$ since this causes no confusion. The following example shows how MR, UDS, and UTS cannot be guaranteedeven with well-behaved distributions - when symmetric dependence is violated.

Example 1 Let $f_{X}$ be constant and positive on $[-11,11]$, let $\tilde{\varepsilon} \mid x \sim U[-1,1](\tilde{\varepsilon} \mid x$ is uniformly distributed on $[-1,1])$ for all $x \geq 0$, and $\tilde{\varepsilon} \mid x \sim U[-11,11]$ for all $x<0$. In this case, $E[X \mid z=0]=-5 / 2$, violating MR, and $E[X \mid z=1]=-7 / 8$, violating both UDS and UTS. Thus, quasiconcavity and symmetry of the random variables is not sufficient for any of these properties without adding symmetric dependence.

Given its clear importance, we assume symmetric dependence for the remainder of this subsection and only work with independent error terms (which therefore satisfy symmetric dependence) in the next subsection.

A4: All error terms $\tilde{\varepsilon}$ satisfy symmetric dependence.

Note that, in particular, we assume that each $\tilde{\varepsilon} \mid x$ has a density; consequently, conditional densities are given by Eq. 1 .

The proofs of the three propositions in this section all make use of the following lemma:

Lemma 1 If $X$ is symmetric and $\tilde{\varepsilon}$ satisfies symmetric dependence, then $E[X \mid z] \gtreqless 0$ if and only if

$$
\int_{0}^{\infty} x\left[f_{\tilde{\varepsilon}}(z-x \mid x)-f_{\tilde{\varepsilon}}(z+x \mid x)\right] f_{X}(x) \mathrm{d} x \gtreqless 0 .
$$

Proof Suppose $E[X \mid z] \geq 0$ (the proofs for $E[X \mid z]=0$ and $E[X \mid z] \leq 0$ are identical). Then

$$
\int_{-\infty}^{\infty} x f_{X \mid Z}(x \mid z) \mathrm{d} x \geq 0
$$

By splitting the integral at zero, this becomes

$$
\int_{0}^{\infty} x f_{X \mid z}(x \mid z) \mathrm{d} x \geq \int_{-\infty}^{0}-x f_{X \mid z}(x \mid z) \mathrm{d} x
$$


or, by a change of variables and rearranging,

$$
\int_{0}^{\infty} x\left[f_{X \mid z}(x \mid z)-f_{X \mid z}(-x \mid z)\right] \mathrm{d} x \geq 0
$$

By Bayes's rule,

$$
\begin{aligned}
f_{X \mid z}(x \mid z) & =\frac{f_{Z \mid x}(z \mid x) f_{X}(x)}{\int f_{Z \mid t}(z \mid t) f_{X}(t) \mathrm{d} t} \\
& =\frac{f_{\tilde{\varepsilon}}(z-x \mid x) f_{X}(x)}{\int f_{Z \mid t}(z \mid t) f_{X}(t) \mathrm{d} t} .
\end{aligned}
$$

Thus, inequality 4 becomes

$$
\int_{0}^{\infty} x\left[f_{\tilde{\varepsilon}}(z-x \mid x) f_{X}(x)-f_{\tilde{\varepsilon}}(z+x \mid-x) f_{X}(-x)\right] \mathrm{d} x \geq 0 .
$$

Symmetry of $X$ implies that $f_{X}(x)=f_{X}(-x)$ and symmetric dependence implies that $f_{\tilde{\varepsilon}}(\varepsilon \mid x)=f_{\tilde{\varepsilon}}(\varepsilon \mid-x)$ for any $x$ and $\varepsilon$. Therefore, Eqs. 3 and 5 are equivalent.

We are now prepared to demonstrate a sufficient condition for the mean reinforcement property.

Proposition 1 If $X$ and $\tilde{\varepsilon}$ are symmetric, then the pair $(X, \tilde{\varepsilon})$ satisfies mean reinforcement $(M R)$.

Proof Without loss of generality assume that $E[X]=0$. Letting $z=0$, we now wish to show that $E[X \mid 0]=0$. Using Lemma 1, this is equivalent to showing that

$$
\int_{0}^{\infty} x\left[f_{\tilde{\varepsilon}}(0-x \mid x)-f_{\tilde{\varepsilon}}(0+x \mid x)\right] f_{X}(x) \mathrm{d} x=0 .
$$

But the bracketed term equals zero almost everywhere since $\tilde{\varepsilon}$ is symmetric, so the claim follows immediately.

The following example shows that these sufficient conditions for MR are not sufficient for the stronger requirement of updating in the direction of the signal (UDS).

Example 2 Consider an $X$ that is uniformly distributed over [-2, 2] and an error term $\tilde{\varepsilon}$ that is independent of $X$ with the symmetric two-point distribution $(-10,1 / 2 ; 10$, 1/2). ${ }^{9}$ If the signal realization is $z=9$, then it is known with certainty that $x=-1$ and $\varepsilon=10$. UDS requires $E[X \mid z=9]>0$, but here $E[X \mid z=9]=-1$, so UDS fails.

\footnotetext{
9 These distributions may be approximated by continuous densities to conform to the assumptions of this subsection.
} 
In order to guarantee UDS, a stronger condition is needed. From Example 2, it is clear that quasiconcavity of the prior is not sufficient. Instead, we find that quasiconcavity of the error distribution is sufficient for UDS, even when the prior is symmetric, but not quasiconcave.

Proposition 2 If $X$ is symmetric and $\tilde{\varepsilon}$ is symmetric and quasiconcave, then the pair $(X, \tilde{\varepsilon})$ satisfies UDS.

Proof Without loss of generality, let $E[X]=0$. Assume first that $z \geq 0$, in which case we wish to show that $E[X \mid z] \geq 0$. Using Lemma 1, this is equivalent to showing that

$$
\int_{0}^{\infty} x\left[f_{\tilde{\varepsilon}}(z-x \mid x)-f_{\tilde{\varepsilon}}(z+x \mid x)\right] f_{X}(x) \mathrm{d} x \geq 0 .
$$

Symmetry of $\tilde{\varepsilon}$ implies that $f_{\tilde{\varepsilon}}(z-x \mid x)=f_{\tilde{\varepsilon}}(|z-x| \mid x)$, so the bracketed term can be rewritten as

$$
\left[f_{\tilde{\varepsilon}}(|z-x| \mid x)-f_{\tilde{\varepsilon}}(z+x \mid x)\right] .
$$

We now show that this bracketed term is non-negative for almost all $x \in[0, \infty)$. First, since $f_{\tilde{\varepsilon}}$ is symmetric and quasiconcave, it is maximized at its mean: $f_{\tilde{\varepsilon}}(0 \mid x) \geq$ $f_{\tilde{\varepsilon}}(\varepsilon \mid x)$ for all $\varepsilon$ and almost every $x$. Second, since $z$ and $x$ are non-negative in this integral, it must be that $0 \leq|z-x| \leq z+x$, and so there is some $\lambda \in(0,1)$ such that $|z-x|=\lambda 0+(1-\lambda)(z+x)$. But since $f_{\tilde{\varepsilon}}(0 \mid x) \geq f_{\tilde{\varepsilon}}(z+x \mid x)$, quasiconcavity implies that $f_{\tilde{\varepsilon}}(|z-x| \mid x) \geq f_{\tilde{\varepsilon}}(z+x \mid x)$ for almost all $x$. Therefore, the bracketed term is non-negative almost everywhere under the integral, and so inequality (6) holds, proving the result for $z \geq 0$. The proof for $z \leq 0$ is symmetric.

We now consider the stronger condition of updating toward the signal (UTS). The following example shows that symmetry of the prior and symmetry and quasiconcavity of the error distribution-which were sufficient for UDS—are not sufficient for UTS.

Example 3 In this example, we simply switch the role of the two distributions in Example 2. Specifically, let $X$ has (a continuous approximation of) the symmetric twopoint distribution $(-10,1 / 2 ; 10,1 / 2)$ and let $\tilde{\varepsilon}$ has the (symmetric and quasiconcave) uniform distribution over $[-2,2]$. Now, when $z=9$, it is known with certainty that $x=10$ and $\varepsilon=-1$. UTS requires that $E[X \mid z=9] \in[0,9]$ but $E[X \mid z=9]=10$, so UTS fails.

Our final sufficiency result shows that UTS is satisfied when both the error distribution and the prior distribution are symmetric and quasiconcave, and in addition, the error term is independent of the prior. It is well known that if the prior and error term are independent and normally distributed, then the pair satisfies UTS; this fact can now be derived as a corollary of the following result. 
Proposition 3 If $X$ is symmetric and quasiconcave and $\tilde{\varepsilon}$ is symmetric, quasiconcave, and independent of $X$, then the pair $(X, \tilde{\varepsilon})$ satisfies UTS.

Proof Without loss of generality let $E[X]=0$ and consider the case where $z \geq 0$. By Proposition 2 we know that UDS is satisfied, so $E[X \mid z] \geq 0$. It remains to show that $E[X \mid z] \leq z$.

Note that since $Z=X+\tilde{\varepsilon}, E[X \mid z] \leq z$ if and only if $E[\tilde{\varepsilon} \mid z] \geq 0$, or

$$
\int_{-\infty}^{\infty} \varepsilon f_{\tilde{\varepsilon} \mid z}(\varepsilon \mid z) \mathrm{d} \varepsilon \geq 0
$$

Breaking the integral at zero, this inequality becomes

$$
\int_{0}^{\infty} \varepsilon f_{\tilde{\varepsilon} \mid z}(\varepsilon \mid z) \mathrm{d} \varepsilon \geq \int_{-\infty}^{0}-\varepsilon f_{\tilde{\varepsilon} \mid z}(\varepsilon \mid z) \mathrm{d} \varepsilon
$$

Applying a change of variables (from $\varepsilon$ to $-\varepsilon$ ) and rearranging gives

$$
\int_{0}^{\infty} \varepsilon\left[f_{\tilde{\varepsilon} \mid z}(\varepsilon \mid z)-f_{\tilde{\varepsilon} \mid z}(-\varepsilon \mid z)\right] \mathrm{d} \varepsilon \geq 0
$$

By Bayes's rule,

$$
f_{\tilde{\varepsilon} \mid z}(\varepsilon \mid z)=\frac{f_{\tilde{\varepsilon}}(\varepsilon \mid z-\varepsilon) f_{X}(z-\varepsilon)}{\int f_{\tilde{\varepsilon}}(t \mid z-t) f_{X}(z-t) \mathrm{d} t},
$$

so the above inequality becomes

$$
\int_{0}^{\infty} \varepsilon\left[f_{\tilde{\varepsilon}}(\varepsilon \mid z-\varepsilon) f_{X}(z-\varepsilon)-f_{\tilde{\varepsilon}}(-\varepsilon \mid z+\varepsilon) f_{X}(z+\varepsilon)\right] \mathrm{d} \varepsilon \geq 0 .
$$

Since $\tilde{\varepsilon}$ is symmetric and independent of $X$, then $f_{\tilde{\varepsilon}}(\varepsilon \mid z-\varepsilon)=f_{\tilde{\varepsilon}}(-\varepsilon \mid z+\varepsilon)$ for every $\varepsilon$ and $z$, so the inequality can be rewritten as

$$
\int_{0}^{\infty} \varepsilon f_{\tilde{\varepsilon}}(\varepsilon)\left[f_{X}(z-\varepsilon)-f_{X}(z+\varepsilon)\right] \mathrm{d} \varepsilon \geq 0
$$

Since $|z-\varepsilon| \leq z+\varepsilon$ for non-negative $\varepsilon$, symmetry and quasiconcavity of $f_{X}$ imply that the bracketed term is non-negative almost everywhere [the proof of this fact follows exactly the same arguments used to show that inequality (6) from the proof Proposition 2 is non-negative almost everywhere]. Thus, the integral is non-negative, proving the result. The proof for $z \leq 0$ is symmetric. 
Table 1 Summary of results

\begin{tabular}{|c|c|c|c|c|c|c|c|}
\hline \multirow{2}{*}{$\frac{\text { Result }}{\text { Proposition } 1}$} & \multicolumn{3}{|c|}{ Error terms } & \multicolumn{3}{|l|}{ Prior } & \multirow{2}{*}{$\begin{array}{l}\text { Condition } \\
\text { MR }\end{array}$} \\
\hline & Sym & & & Sym & & $\Rightarrow$ & \\
\hline Example 2 & Sym & & Ind & Sym & QC & $\nRightarrow$ & UDS \\
\hline Proposition 2 & Sym & $\mathrm{QC}$ & & Sym & & $\Rightarrow$ & UDS \\
\hline Example 3 & Sym & $\mathrm{QC}$ & Ind & Sym & & $\nRightarrow$ & UTS \\
\hline Example 4 & Sym & $\mathrm{QC}$ & & Sym & QC & $\not$ & UTS \\
\hline Proposition 3 & Sym & $\mathrm{QC}$ & Ind & Sym & QC & $\Rightarrow$ & UTS \\
\hline Proposition 4 & \multicolumn{3}{|c|}{ All Ind, Sym, 2-Point Errors } & Sym & & $\Leftarrow$ & MR \\
\hline Proposition 5 & \multicolumn{3}{|c|}{ All Ind, Sym, 2-Point Errors } & \multicolumn{2}{|c|}{$\nexists$} & $\Leftarrow$ & UDS \\
\hline Proposition 6 & \multicolumn{3}{|c|}{ All Ind Uniform Errors } & Sym & & $\Leftarrow$ & MR \\
\hline Proposition 7 & \multicolumn{3}{|c|}{ All Ind Uniform Errors } & Sym & QC & $\Leftarrow$ & UTS \\
\hline
\end{tabular}

Sym symmetric, $Q C$ quasiconcave, Ind independent

Unlike the previous results, Proposition 3 requires independent error terms. The following example shows that if independence is relaxed to symmetric dependence, then the UTS no longer holds.

Example 4 Let $X \sim U[-4,4]$. If $|x| \leq 1$, then let $\tilde{\varepsilon} \mid x \sim U[-1,1]$ and if $|x|>1$, then $\tilde{\varepsilon} \mid x \sim U[-2,2]$. All random variables are symmetric and quasiconcave, and $\tilde{\varepsilon} \mid x$ satisfies symmetric dependence but is not independent of $X$. Consider $z=2$, where $f_{Z}(2)=\int_{-4}^{4} f_{\tilde{\varepsilon}}(z-x \mid x) f_{X}(x) \mathrm{d} x$ equals $\int_{1}^{4}(1 / 4)(1 / 8) \mathrm{d} x=3 / 32$. Thus, $E[X \mid z=2]$ equals $\int_{-4}^{4} x f_{\tilde{\varepsilon}}(z-x \mid x) f_{X}(x) /(3 / 32) \mathrm{d} x$, or $\int_{1}^{4} x(1 / 4)(1 / 8) /(3 / 32) \mathrm{d} x=$ $\int_{1}^{4} x \mathrm{~d} x(1 / 3)$, which equals $5 / 2$. Since $E[X \mid z=2]>2$, UTS fails.

A summary of the sufficiency results appears in the top half of Table 1 . The boundaries of Proposition 3 are nicely highlighted by comparing it to Examples 2, 4, and 3 ; if we relax quasiconcavity of the prior or independence of the error then UTS fails (Examples 3 and 4), and if we relax quasiconcavity of the error terms then both UTS and UDS fail (Example 2).

\subsection{Necessary conditions}

We now explore conditions on the prior and error distributions that must be true of the pair satisfies MR, UDS, and UTS. The following example shows, however, that no combination of symmetry and quasiconcavity is necessary for MR, UDS, or UTS.

Example 5 Let $X$ and $\tilde{\varepsilon}$ both have (continuous approximations of) the two-point distribution $(4,1 / 3 ;-2,2 / 3)$. This mean-zero distribution is neither symmetric nor quasiconcave though $\tilde{\varepsilon}$ is independent of $X$ and, therefore, satisfies symmetric dependence. The three possible signal realizations are $z=8, z=2$, and $z=-4$. If $z \in\{8,-4\}$, then $x$ is known with certainty. In particular, $E[X \mid z=8]=4$ and $E[X \mid z=-4]=-2$. If $z=2$ is observed, then $x=4$ and $x=-2$ are equally likely; thus, $E[X \mid z=2]=1$. 
In all three cases, $E[X \mid z]=z / 2$, so UDS and UTS are satisfied, and MR is vacuously satisfied.

Given this difficulty in identifying necessary conditions for any particular pair $(X, \tilde{\varepsilon})$, we follow Milgrom (1981) and ask what conditions MR, UTS, and UDS would imply on one of the distributions given freedom in the specification of the other. Specifically, we take the viewpoint of a modeler who fixes a prior distribution but, in the interest of model robustness, is unwilling to commit to a particular error distribution. For example, she may select a normally distributed prior but allow for any symmetric and quasiconcave error distribution. In this setting, we ask what the restrictions on the prior must be if MR, UDS, or UTS is assumed to hold for all such error distributions.

We focus on two cases of interest: First, when the modeler requires MR, UDS, or UTS to hold for all symmetric, independent, two-point error distributions (or, by extension, any larger family of error distributions that includes all symmetric, twopoint distributions) and second, when the modeler requires MR, UDS, or UTS to hold for all independent, uniformly distributed error terms (or any larger family).

Our interpretation of these two families of error distributions is that any sufficiently broad family of error terms that includes symmetric (but not necessarily quasiconcave) distributions will include all symmetric, independent, two-point error distributions as a subset, and any sufficiently broad family of error terms that includes symmetric and quasiconcave distributions will include all independent, uniformly distributed error terms as a subset. The results of this section explore the freedom in the choice of the prior that can be obtained when one requires MR, UDS, or UTS while allowing for such flexibility in the specification of the error terms.

Clearly, studying two-point or uniformly distributed error terms violates the symmetric dependence assumption because symmetric dependence - as defined aboverequires $\tilde{\varepsilon}$ to have a continuous density function. We dispose with this assumption and work only with error terms that are independent of $X$. Our results then apply to any family containing these families of independent error terms. We link our sufficiency results (which assume symmetric dependence) with these necessity results in the next subsection.

Proposition 1 showed that symmetry of both the error and prior distribution is sufficient for mean reinforcement (MR). The following provides a rough converse to this result: if all symmetric two-point distributions are admissible, then requiring MR for all admissible error terms implies that the prior distribution must be symmetric.

Proposition 4 If $X$ is such that $(X, \tilde{\varepsilon})$ satisfies mean reinforcement $(M R)$ for all independent, symmetric, error terms $\tilde{\varepsilon}$ with a two-point distribution, then $X$ is symmetric.

Proof We prove the contrapositive, which says that if $X$ is not symmetric then there is some two-point error term for which MR fails.

Assume without loss of generality that $E[X]=0$. Since $X$ is not symmetric, there is some $y>0$ such that $f_{X}(y) \neq f_{X}(-y)$. Consider the error term $\tilde{\varepsilon}$ with the twopoint distribution $(-y, 1 / 2 ; y, 1 / 2)$, which is independent of $X$. The posterior mean given $z=0$ is then

$$
E[X \mid z=0]=\frac{y f_{X}(y)-y f_{X}(-y)}{f_{X}(y)+f_{X}(-y)},
$$


which cannot equal zero (the prior mean) since $f_{X}(y) \neq f_{X}(-y)$ (see Fig. 1a for an illustration). Thus, MR fails.

With a broad enough class of symmetric error terms, the above result shows that mean reinforcement implies symmetry of the prior. We now demonstrate that one cannot use the stronger condition of UDS if this same family of error terms is admissible.

Proposition 5 There is no $X$ such that $(X, \tilde{\varepsilon})$ satisfies UDS for all independent, symmetric error terms $\tilde{\varepsilon}$ with a two-point distribution.

Proof Suppose, by way of contradiction, that $X$ satisfies UDS for all independent, symmetric $\tilde{\varepsilon}$ with two-point distributions. Pick any pair $\left(x_{1}, x_{2}\right)$ with $x_{2}>x_{1}>0$ and consider the error term

$$
\tilde{\varepsilon}_{\left(x_{1}, x_{2}\right)} \sim\left(-\frac{x_{1}+x_{2}}{2}, \quad \frac{1}{2} ; \frac{x_{1}+x_{2}}{2}, \frac{1}{2}\right)
$$

and the signal $z=\left(x_{2}-x_{1}\right) / 2$ when $Z=X+\tilde{\varepsilon}_{\left(x_{1}, x_{2}\right)}$. In this case, the posterior on $X$ given $z$ has the two-point distribution

$$
\left(-x_{1}, \frac{f_{X}\left(-x_{1}\right)}{f_{X}\left(-x_{1}\right)+f_{X}\left(x_{2}\right)} ; \quad x_{2}, \frac{f_{X}\left(x_{2}\right)}{f_{X}\left(-x_{1}\right)+f_{X}\left(x_{2}\right)}\right) \text {. }
$$

Thus, $E[X \mid z]$ is proportional to $-x_{1} f_{X}\left(-x_{1}\right)+x_{2} f_{X}\left(x_{2}\right)$, which must be non-negative since $X$ satisfies UDS. Therefore, $x_{2} f_{X}\left(x_{2}\right) \geq x_{1} f_{X}\left(-x_{1}\right)$. Since UDS implies MR, we know by Proposition 4 that $X$ must be symmetric, so we have that

$$
x_{2} f_{X}\left(x_{2}\right) \geq x_{1} f_{X}\left(x_{1}\right) \text {. }
$$

But this argument can be repeated for all $x_{2}>x_{1}>0$, so $x f_{X}(x)$ is increasing in $x$ for $x>0$. But then $E[X]=\int_{-\infty}^{\infty} x f_{X}(x) \mathrm{d} x$ does not exist unless $f_{X}$ is zero almost everywhere. This is a contradiction since UDS requires that $X$ have a finite mean.

The above impossibility result implies that UDS and UTS cannot be satisfied if all independent, symmetric, two-point error distributions are admissible. The sufficiency results, however, indicate that quasiconcavity also plays a role in both UDS and UTS, so we proceed by adding quasiconcavity to the admissible family of error terms. This is done by considering the family of all independent, uniformly distributed error terms. We now show that in this case symmetry of $X$ is not only implied by UDS, but that it is implied by the weaker concept of mean reinforcement as well. Note that the following Proposition relies on error terms possessing densities, so that Eq. 1 is used for computing conditional expectations.

Proposition 6 If $X$ is such that $(X, \tilde{\varepsilon})$ satisfies mean reinforcement $(M R)$ for all independent, uniformly distributed error terms $\tilde{\varepsilon}$, then $X$ is symmetric.

Proof Suppose that $(X, \tilde{\varepsilon})$ satisfies MR for every independent, uniformly distributed error term $\tilde{\varepsilon}$. Pick any $y>0$ and choose $\tilde{\varepsilon}_{y}$ to have the uniform distribution over 
$[-y, y]$. In this case, the posterior mean given $z=0$ is proportional to $\int_{-y}^{y} x f_{X}(x) \mathrm{d} x$. If $\left(X, \tilde{\varepsilon}_{y}\right)$ satisfies MR for every $y>0$, then

$$
\int_{-y}^{y} x f_{X}(x) \mathrm{d} x=0
$$

for almost every $y>0$. Differentiating this expression with respect to $y$ establishes that

$$
y f_{X}(y)=y f_{X}(-y)
$$

or

$$
f_{X}(y)=f_{X}(-y)
$$

for almost every $y>0$. Thus, $X$ is symmetric.

Finally, we saw from the sufficiency results that quasiconcavity of the prior can be used to guarantee UTS, so we demonstrate now that UTS implies that the prior should also be quasiconcave when all independent, uniform distributions are admissible.

Proposition 7 If $X$ is such that $(X, \tilde{\varepsilon})$ satisfies UTS for all independent, uniformly distributed error terms $\tilde{\varepsilon}$, then $X$ is symmetric and quasiconcave.

Proof Proposition 6 and the fact that UTS implies MR give the result that $X$ is symmetric; we now demonstrate a failure of UTS when $X$ is symmetric, but not quasiconcave.

Let $E[X]=0$ (without loss of generality) and consider any $z \geq 0$. UTS implies that $E[X \mid z] \leq z$, or equivalently that $E[\tilde{\varepsilon} \mid z] \geq 0$. Consider the error term $\tilde{\varepsilon}$ with a uniform distribution over $[-y, y]$. Then $E[\tilde{\varepsilon} \mid z] \geq 0$ if and only if

$$
\int_{-y}^{y} \varepsilon f_{X}(z-\varepsilon) \mathrm{d} \varepsilon \geq 0
$$

By breaking this integral at $y=0$ and applying a change of variables the expression can be rewritten as

$$
\int_{0}^{y} \varepsilon\left[f_{X}(z-\varepsilon)-f_{X}(z+\varepsilon)\right] \mathrm{d} \varepsilon \geq 0 .
$$

Since $f_{X}$ is not quasiconcave then, by symmetry, there is a pair of values $x^{\prime \prime}>$ $x^{\prime} \geq 0$ such that $f_{X}\left(x^{\prime \prime}\right)>f_{X}\left(x^{\prime}\right)$. Fix some $\alpha \in\left(f_{X}\left(x^{\prime}\right), f_{X}\left(x^{\prime \prime}\right)\right)$. Let $\hat{x}=\sup \{x \in$ $\left.\left[x^{\prime}, x^{\prime \prime}\right]: f_{X}(x)<\alpha\right\}$ so that $f_{X}(\hat{x})=\alpha$ by continuity. Now let $x^{*}=\inf \{x \geq \hat{x}$ : $f_{X}(x)>\alpha$ so that $f_{X}\left(x^{*}\right)=\alpha$ as well though it may be the case that $x^{*}>\hat{x}$. 
Since $\alpha>f_{X}\left(x^{\prime}\right)$ it must be that $\hat{x}>x^{\prime} \geq 0$, and so $x^{*}>0$. Thus, there is some $\delta>0$ small enough such that $x^{*}-\delta>0$ and, for each $\varepsilon \in(0, \delta), f_{X}\left(x^{*}-\varepsilon\right) \leq \alpha$ and $f_{X}\left(x^{*}+\varepsilon\right)>\alpha$. We have therefore established an interval $\left(x^{*}-\delta, x^{*}+\delta\right)$ with $f_{X} \leq \alpha$ on the lower half of the interval and $f_{X}>\alpha$ on the upper half. Thus, $f_{X}\left(x^{*}+\varepsilon\right)>f_{X}\left(x^{*}-\varepsilon\right)$ for all $\varepsilon \in(0, \delta)$ (see Fig. 1b for an illustration). But if we consider the case where the signal realization is $z=x^{*}$ and the error term is uniformly distributed on $[-y, y]$ with $y=\delta$, then UTS implies Eq. 9, which contradicts the fact that $f_{X}(z+\varepsilon)>f_{X}(z-\varepsilon)$ for almost every $\varepsilon$ in $[0, y]$. Thus, UTS fails. The proof for $z \leq 0$ is symmetric.

\subsection{Characterizations}

All sufficiency and necessity results are summarized in Table 1. Combining each sufficiency result with the appropriate necessity result allows us to state three characterization theorems that roughly summarize the findings throughout the paper.

The first characterization highlights the link between mean reinforcement and symmetry of the prior and error distributions.

Corollary $1 X$ is symmetric if and only if $(X, \tilde{\varepsilon})$ satisfies $M R$ for every independent, symmetric, two-point error term $\tilde{\varepsilon}$.

Proof Necessity follows immediately from Proposition 4. For sufficiency, we cannot directly apply Proposition 1 because its proof required $\tilde{\varepsilon}$ to have a well-defined density. However, sufficiency is easily proven directly: Without loss, assume $X$ is symmetric with $E[X]=0$, and let $\tilde{\varepsilon}$ has two-point distribution $(-y, 1 / 2 ; y, 1 / 2)$, independent of $X$. Then $E[X \mid z=0]$ is proportional to $y f_{X}(y)-y f_{X}(-y)$, which equals zero by symmetry of $f_{X}$, and so MR is satisfied.

The second characterization is perhaps the most surprising; we show that MR is exactly as strong as UDS when the family of admissible error terms is a broad enough family of symmetric and quasiconcave errors.

Corollary 2 Consider the following three statements:

1. $(X, \tilde{\varepsilon})$ satisfies $M R$ for all independent, uniformly distributed error terms $\tilde{\varepsilon}$,

2. $X$ is symmetric, and

3. $(X, \tilde{\varepsilon})$ satisfies UDS for all independent, uniformly distributed error terms $\tilde{\varepsilon}$.

We have that $(1) \Leftrightarrow(2) \Rightarrow(3)$. If $E[X \mid z]$ is continuous at $z=E[X]$, then all three statements are equivalent.

Proof Statement (1) implies (2) by Proposition 6, and (2) implies (1) by Proposition 1 since uniform distributions are symmetric. Statement (2) also implies (3) by Proposition 2 since uniform distributions are also quasiconcave. If $E[X \mid z]$ is continuous at $z=E[X]$ then UDS implies MR, and so statement (3) implies (1).

Finally, we find an equivalence between priors that satisfy UTS for all independent, uniformly distributed error terms and priors whose distributions are symmetric and quasiconcave. 
Corollary $3 X$ is symmetric and quasiconcave if and only if $(X, \tilde{\varepsilon})$ satisfies UTS for all independent, uniformly distributed error terms $\tilde{\varepsilon}$.

Proof Follows immediately from Propositions 3 and 7.

\section{Application: portfolio selection}

We now demonstrate our main theorems by applying them to the well-known portfolio allocation problem described by Arrow (1971, Chapter 3). ${ }^{10}$ Consider a risk-averse investor allocating a fixed endowment of wealth between a risky asset and a riskfree asset. Initially, he makes a portfolio allocation decision based only on his prior beliefs about the risky asset's return. Then he receives an informative signal and is asked if he would like to revise his investment decision. In this setting, Observation 1 shows that an outside observer who knows nothing about the shape of the investor's belief distributions can never predict whether and in what direction the investor will switch his investment strategy after observing the signal. This is true even if the utility function, risk-free return, signal realization and prior mean are all public information.

It may seem fairly innocuous to assume, however, that an investor who has chosen to invest a positive amount in the risky asset before observing a signal will continue to invest some positive amount in it if the signal is, in some sense, favorable. Similarly, one might assume that investors who do not invest in (or who sell) the risky asset continue not investing (or selling) after seeing unfavorable signals. Given Observation 1, such assumptions place some restriction on the underlying belief distributions, but it is not at all transparent how severe a restriction they imply.

To formalize the argument, let the agent's endowment of wealth be given by $\omega>0$, and his utility for money be a twice-differentiable function $u: \mathbb{R} \rightarrow \mathbb{R}$ with $u^{\prime}>0$ and $u^{\prime \prime} \leq 0$. The return on the risk-free asset is $\hat{r} \geq 1$, which is known with certainty, and the return on the risky asset is given by the random variable $X$ with distribution function $F_{X}$ and mean $\mu_{X}$. A typical realization of $X$ is denoted by $x$.

The signal shown to the investor is a realization $z$ of the random variable $Z:=X+\tilde{\varepsilon}$, where $\tilde{\varepsilon}$ is a mean-zero error term with distribution $F_{\tilde{\varepsilon}}$. In this section, we use only error terms $\tilde{\varepsilon}$ that are independent of $X$. Using Bayes's rule, the investor's posterior belief about $X$ upon observing $Z=z$ is given by the distribution $F_{X \mid Z}(\cdot \mid z)$.

From a modeling perspective, we will take the prior distribution $X$ as fixed. We consider the case where $F_{\tilde{\varepsilon}}$ might be one of any number of possible error distributions. For example, the signal might represent an early earnings report that, though unbiased, is believed to be more informative by some investors than by others. In the interest of model robustness, one might want to allow for the possibility that all independent and uniformly distributed error distributions are admissible. Thus, assuming that positive signals always reinforce positive investments in the risky asset means assuming this property for all admissible error terms $\tilde{\varepsilon}$. The larger the family of admissible error terms, the stronger such assumptions become.

\footnotetext{
10 See also Markowitz (1952), Markowitz (1958), Pratt (1964), and Tobin (1958) for further discussion of the basic portfolio selection problem.
} 
The investor's choice before receiving a signal is to invest an amount $y_{0} \in \mathbb{R}$ in the risky asset (assuming no credit constraints), with $\omega-y_{0}$ being invested in the risk-free asset. Upon observing the signal realization, the investor forms updated beliefs and re-solves his optimization problem, resulting in a new investment level $y_{1}$. Formally, the pre-signal investment level is the maximizer of the function

$$
V\left(y_{0}\right):=\int u\left(\omega \hat{r}+y_{0}[x-\hat{r}]\right) \mathrm{d} F_{X}(x)
$$

and the post-signal investment (given $Z=z$ ) maximizes

$$
V\left(y_{1} \mid z\right):=\int u\left(\omega \hat{r}+y_{1}[x-\hat{r}]\right) \mathrm{d} F_{X \mid Z}(x \mid z) .
$$

Here, $[x-\hat{r}]$ is referred to as the excess return of the risky asset. For simplicity, we say that the investor "invests" prior to the signal if $y_{0}^{*}>0$ and "does not invest" if $y_{0}^{*} \leq 0$, and that he "invests" after the signal if $y_{1}^{*}>0$ and "does not invest" if $y_{1}^{*} \leq 0$.

We now define three different notions of how the investor's signal might reinforce his prior investment decision.

Definition 7 The investor's decision is reinforced at the prior mean if, for all $\hat{r}$, he never changes his decision $\left(y_{1}^{*}=y_{0}^{*}\right)$ after observing $Z=E[X]$.

Definition 8 The investor's decision is reinforced around the prior mean if, for all $\hat{r}$, whenever he has invested $\left(y_{0}^{*}>0\right)$ and then receives a signal above the prior mean $(z>E[X])$ he continues to invest $\left(y_{1}^{*}>0\right)$, and whenever he has not invested $\left(y_{0}^{*} \leq 0\right)$ and then receives a signal below the prior mean $(z<E[X])$ he continues not to invest $\left(y_{1}^{*} \leq 0\right)$.

Definition 9 The investor's decision is reinforced around $\hat{r}$ if, for all $\hat{r}$, whenever he has invested $\left(y_{0}^{*}>0\right)$ and then receives a signal above the risk-free return $(z>\hat{r})$ he continues to invest $\left(y_{1}^{*}>0\right)$, and whenever he has not invested $\left(y_{0}^{*} \leq 0\right)$ and then receives a signal below the risk-free return $(z<\hat{r})$ he continues not to invest $\left(y_{1}^{*} \leq 0\right)$.

Applying two of our main results gives the following characterizations.

Theorem 1 If $E[X \mid z]$ is continuous at $z=E[X]$, then the following are equivalent:

1. The investor's decision is reinforced by the mean signal for all uniformly distributed error terms $\tilde{\varepsilon}$.

2. The investor's decision is reinforced around the prior mean for all uniformly distributed error terms $\tilde{\varepsilon}$.

3. The investor's prior distribution has a symmetric density function.

Theorem 2 The investor's prior has a symmetric and quasiconcave density function if and only if his decision is reinforced around $\hat{r}$ for all independent, uniformly distributed error terms $\tilde{\varepsilon}$.

Thus, a modeler who assumes that good signals always reinforce a decision to invest (for all uniform error distributions) and bad signals always reinforce a decision not to invest implicitly assumes symmetry and perhaps quasiconcavity of the prior distribution. 


\section{Appendix: Proofs of Theorems 1 and 2}

We first show the equivalence between MR, UDS, and UTS and Definitions 7-9, respectively.

Proposition 8 An investor's beliefs satisfy MR if and only if his decision is reinforced at the prior mean, his beliefs satisfy UDS if and only if his decision is reinforced around the prior mean, and his beliefs satisfy UTS if and only if his decision is reinforced around $\hat{r}$.

This follows from a well-known proposition in the portfolio choice literature:

Lemma 2 A risk-averse agent invests a positive (negative) amount in the risky asset if and only if the agent's expected excess return for the risky asset is positive (negative).

Proof The first-order condition for pre-signal maximization at some optimal point $y_{0}^{*}$ is given by

$$
V^{\prime}\left(y_{0}^{*}\right)=\int[x-\hat{r}] u^{\prime}\left(\omega \hat{r}+y_{0}^{*}[x-\hat{r}]\right) \mathrm{d} F_{X}(x)=0 .
$$

The objective function $V$ is weakly concave in $y_{0}$ since $u^{\prime \prime} \leq 0$, so the first-order condition is also sufficient. Note that at $y_{0}=0$,

$$
\begin{aligned}
V^{\prime}(0) & =u^{\prime}(\omega \hat{r}) \int[x-\hat{r}] \mathrm{d} F_{X}(x) \\
& =u^{\prime}(\omega \hat{r})[E[X]-\hat{r}],
\end{aligned}
$$

so $V^{\prime}(0)$ has the same sign as $E[X]-\hat{r}$. Thus, if $E[X]>\hat{r}$ then $V^{\prime}(0)>0$ and so he invests a positive amount in the risky asset. If $E[X]<\hat{r}$, then the optimal investment quantity is negative. Identical arguments establish that $y_{1}^{*}>0$ if $E[X \mid Z=z]>\hat{r}$ and $y_{1}^{*}<0$ if $E[X \mid Z=z]<\hat{r}$.

Proof (Sketch of the Proof for Proposition 8) For example, suppose the investor's beliefs satisfy UTS. If he invests before the signal (meaning $E[X]>\hat{r}$ ) and gets a signal above the risk-free return $(z>\hat{r})$, then we must have $E[X \mid Z=z]>\hat{r}$ since, by UTS, $E[X \mid Z=z]$ must be between $E[X]$ and $z$. Thus, he continues to invest. If he does not invest before the signal $(E[X]<\hat{r})$ and $z<\hat{r}$, then $E[X \mid Z=z]<\hat{r}$, and so he continues not to invest. In the other direction, if his beliefs do not satisfy UTS, then there is some $z$ (say, with $z \geq E[X]$ ) such that either $E[X \mid Z=z]<E[X] \leq z$ or $E[X] \leq z<E[X \mid Z=z]$. In the first case, if $\hat{r}$ is such that $E[X \mid Z=z]<\hat{r}<$ $E[X] \leq z$, then the agent invests before the signal, receives a signal above $\hat{r}$, but then does not invest. In the second case, if $\hat{r}$ is such that $E[X] \leq z<\hat{r}<E[X \mid Z=z]$, then the agent does not invest before the signal, receives a signal below $\hat{r}$, but then invests. In either case, his decision is not reinforced by $\hat{r}$. The argument is symmetric for $z \leq E[X]$ ). Finally, similar arguments can be used for the equivalences between MR and decisions reinforced at the prior mean and between UDS and decisions reinforced around the prior mean. 
Given Proposition 8, Theorems 1 and 2 then follow directly from Corollaries 2 and 3 , respectively.

Open Access This article is distributed under the terms of the Creative Commons Attribution Noncommercial License which permits any noncommercial use, distribution, and reproduction in any medium, provided the original author(s) and source are credited.

\section{References}

Aït-Sahalia, V., Mykland, P.A., Zhang, L.: How often to sample a continuous-time process in the presence of market microstructure noise. Rev Financ Stud 18(2), 351-416 (2005)

Andersen, T.G., Bollerslev, T., Diebold, F.X., Labys, P.: Great realizations. Risk 13(3), 105-108 (2000)

Andrews, R.L., Arnold, J.C., Krutchkoff, R.G.: Shrinkage of the posterior mean in the normal case. Biometrika 59(3), 693-695 (1972)

Arrow, K.J.: Essays in the Theory of Risk Bearing. Chicago: Markham (1971)

Azrieli, Y., Lehrer, E.: Market games in large economies with a finite number of types. Econ Theory 31, 327342 (2007)

Berger, J.O.: An overview of robust bayesian analysis. Test 3, 5-124 (1994)

Chambers, C.P., Healy, P.J.: On the robustness of good news and bad news, Ohio State University Working Paper (2009)

DeGroot, M.H.: Some problems of optimal stopping. J R Stat Soc Ser B 30(1), 108-122 (1968)

Diaconis, P., Ylvisaker, D.: Conjugate priors for exponential families. Ann Stat 7(2), 269-281 (1979)

Feldman, M.: On the generic nonconvergence of bayesian actions and beliefs. Econ Theory 1, 301321 (1991)

Ghirardato, P.: Revisiting savage in a conditional world. Econ Theory 20, 83-92 (2002)

Grossman, S., Stiglitz, J.E.: Information and competitive price systems. Am Econ Rev 66, 246-253 (1976)

Grossman, S., Stiglitz, J.E.: On the impossibility of informationally efficient markets. Am Econ Rev 70, 393408 (1980)

Hansen, P.R., Lunde, A.: Realized variance and market microstructure noise. J Bus Econ Stat 24(2), 127161 (2006)

Jackson, M.O., Peck, J.: Asymmetric information in a competitive market game: reexamining the implications of rational expectations. Econ Theory 13, 603-628 (1999)

Kahn, P.M.: Credibility: Theory and Application. New York: Academic Press (1975)

Lucas, R.: Expectations and the neutrality of money. J Econ Theory 4, 103-124 (1972)

Markowitz, H.M.: Portfolio selection. J Financ 7, 77-91 (1952)

Markowitz, H.M.: Portfolio Selection: Efficient Diversification of Investments. New York: Wiley (1958)

Menon, N., Subramanian, N.: Learning, diversification and the nature of risk. Econ Theory 35, 117145 (2008)

Milgrom, P.R.: Goods news and bad news: representation theorems and applications. Bell J Econ 12, 380391 (1981)

Milgrom, P.R., Weber, R.J.: A theory of auctions and competitive bidding. Econometrica 50, 1089$1122(1982)$

Moore, D.A., Healy, P.J.: The trouble with overconfidence. Psychol Rev 115(2), 502-517 (2008)

Morris, S., Shin, H.S.: Social value of public information. Am Econ Rev 92, 1521-1534 (2002)

Morris, S., Shin, H.S.: Beauty contests and iterated expectations in asset marekts. Rev Financ Stud 19, 719_ 752 (2006)

Novshek, W., Sonnenschein, H.: Fulfilled expectations cournot duopoly with information acquisition and release. Bell J Econ 13, 214-218 (1982)

Polson, N.G.: A representation of the posterior mean for a location model. Biometrika 78(2), 426-430(1991)

Pratt, J.W.: Risk aversion in the small and large. Econometrica 32, 122-136 (1964)

Quint, D.: Looking smart versus playing dumb in common-value auctions. Econ Theory 44, 469-490 (2010)

Rothschild, M.: Searching for the lowest price when the distribution of prices is unknown. J Polit Econ 82, 689-711 (1974)

Sandroni, A.: Efficient markets and bayes' rule. Econ Theory 26, 741-764 (2005) 
Shapiro, C.: Exchange of cost information in oligopoly. Rev Econ Stud 53(3), 433-446 (1986)

Tadelis, S.: Firm reputation with hidden information. Econ Theory 21, 635-651 (2003)

Tobin, J.: Liquidity preference as behavior towards risk. Rev Econ Stud 25(2), 65-86 (1958)

Wilson, R.: A bidding model of perfect competition. Rev Econ Stud 44, 511-518 (1977)

Zhang, L., Mykland, P.A., Ait-Sahalia, Y.: A tale of two time scales: Determining integrated volatility with noisy high-frequency data. J Am Stat Assoc 100(472), 1394-1411 (2005)

Zhou, B.: High-frequency data and volatility in foreign-exchange rates. J Bus Econ Stat 14(1), 45-52 (1996) 